\title{
Research Paper \\ Investigating GJB2 Mutation in 31 Individuals With Non-syndromic Hearing Loss
}

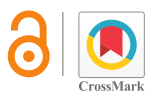

\author{
Pedram Pouryari Biyachal ${ }^{1}\left(\mathbb{0},{ }^{*}\right.$ Najmeh Ranji $^{2}$ 이, Ali Nazemi $^{1}$ (1) \\ 1. Department of Genetics, Faculty of Biological Sciences, Tonekabon Branch, Islamic Azad University, Tonekabon, Iran. \\ 2. Department of Biology, Faculty of Sciences, Rasht Branch, Islamic Azad University, Rasht, Iran.
}

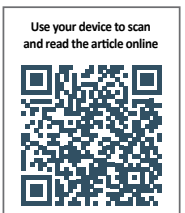

Citation: Pouryari Biyachal P, Ranji N, Nazemi A. [Investigation of GJB2 Mutation in 31 Individuals With Non-syndromic Hearing Loss in Guilan Province (Persian)]. Journal of Arak University of Medical Sciences (JAMS). 2021; 24(1):50-61. https:// doi.org/10.32598/JAMS.24.1.4277.6

d $)^{\prime}$ https://doi.org/10.32598/JAMS.24.1.4277.6

Keywords:

GJB2 gene, Gene

deletion, Hearing loss,

Sequencing

\section{A B S TRACT}

Article Info:

Received: 13 Jul 2020

Accepted: 26 Oct 2020

Available Online: 01 April 2021

\section{Extended Abstract}

\section{Introduction}

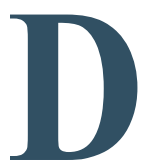

eafness is among the major sensoryneurological deficits; the World Health Organization (WHO) estimates that approximately 360 million individuals with mild to severe deafness live in the world.

Deafness is a heterogeneous disorder, i.e., caused by environmental and genetic factors [1]. Despite the genetic diversity of deafness in different populations, the DFNB1 locus is the cause of approximately $50 \%$ of non-syndromic deafness [2]. The GJB2 gene is located at the DFNB1 locus and has two exons [1]; it produces a protein product called connexin 26 [2]. Connexin 26 acts as an essential regulator of potassium ion $(\mathrm{K}+)$ homeostasis in the inner ear. Without $\mathrm{K}+$ exchanges, auditory cells fail to respond to sound responses [2]. This study aimed to evaluate GJB2 gene mutations by PCR-sequencing in 31 non-syndromic deaf individuals.

\section{Materials and Methods}

In this descriptive cross-sectional study, a total of 85 unrelated deaf individuals from 85 families who were referred

\section{* Corresponding Author: \\ Najmeh Ranji, PhD.}

Address: Department of Biology, Faculty of Sciences, Rasht Branch, Islamic Azad University, Rasht, Iran.

Tel: +98 (13) 33424080

E-mail:n_ranji@iaurasht.ac.ir 
Table 1. Mutations observed in GJB2 gene in the explored deaf subjects

\begin{tabular}{|c|c|c|c|c|c|}
\hline $\begin{array}{l}\text { The Number of } \\
\text { Patients }\end{array}$ & $\begin{array}{c}\text { The Name of the } \\
\text { Mutation }\end{array}$ & Type of Mutation & $\begin{array}{l}\text { Number of Al- } \\
\text { leles }\end{array}$ & Relative Frequency (\%) & Game Changes \\
\hline 16 & c.35delG p.G12VfsX2 & $\begin{array}{l}\text { Removal of a single } \\
\text { nucleotide by changing the } \\
\text { reading format }\end{array}$ & 30 & 48.38 & $\begin{array}{l}\mathrm{GGT}>\mathrm{GTG} \\
\mathrm{CCA}>\mathrm{CAC}\end{array}$ \\
\hline 1 & c.457G>A p.V153I & Meaningful displacement & 1 & 1.61 & GTC $>$ ATC \\
\hline 1 & c.598G> A p.G200R & $\begin{array}{l}\text { Meaningful displacement } \\
\text { (pathogenic variant) }\end{array}$ & 1 & 1.16 & GGA>AGA \\
\hline Total & - & - & 32 & 51.6 & \\
\hline
\end{tabular}

to Guilan General Welfare Office and Rasht Welfare Office, Rasht Deafness Center, and White Silence Deafness Center (in Anzali port) in Gilan Province, Iran, were identified. Among them, 31 patients with non-syndromic deafness and bilateral deafness, deafness from birth, and having one or more deaf relatives, ranging in age from 17 to 50 years were selected. Accordingly, the necessary blood samples were collected from them. After DNA purification, the entire length of the GJB2 gene was amplified by PCR. Besides, sequencing was performed with the reverse primer. The results of sequencing using CLC main workbench v3.5 software and online blast software (BLAST) were examined for mutations in deaf subjects, compared to the reference gene available at the NCBI site.

Ethical considerations: With the permission of the Ethics Committee of the Islamic Azad University, Tonekabon Branch (Code: IR.IAU.TON.REC.1398.015) and with the consent of the deaf subjects or their parents, the sampling of deaf individuals was performed.

\section{Results}

In this study, 31 non-syndromic deaf individuals (out of 85 deaf subjects) from Gilan Province were included; of them, 22(71\%) and 9(29\%) subjects were females and males, in sequence. The mean age of the examined deaf subjects was 33.33 years. In this study, 35 delG mutations in the GJB2 gene were detected in 16 patients (Table 1). Of them, 14 patients were homozygous (Figure 1) and two patients were heterozygous (Figure 2). In one patient, the V153I mutation was observed as heterozygous. In one of two patients with a $35 \mathrm{delG}$ heterozygous mutation, the pathogenic variant of G200R was also observed as heterozygous (35delG/G200R).

\section{Discussion and Conclusion}

Approximately $52 \%$ of the explored deaf individuals from Rasht and Bandar Anzali deaf centers presented mutations in the GJB2 gene; the most common mutation among these individuals was $35 \mathrm{delG}$ mutation with a frequency of $48.38 \%$. Moreover, $35 \mathrm{delG}$ mutation in some studies in Isfahan with a frequency of $6.12 \%$ [1], in the Kurdish population with an allelic frequency of $13.33 \%$ [14], in Qom with a prevalence of $61.9 \%$ [15], in Tabriz with a frequency of $39 \%$ [11], and Lorestan with a frequency of 9.4\% [16] was reported. The present study was more similar to Tabriz and Qom data concerning the frequency of $35 \mathrm{delG}$ mutation. However, in some parts of Iran, including Kurdistan, Azerbaijan, Isfahan, and neighboring countries (Turkey \& Pakistan), the prevalence of this mutation is lower.

The allelic frequency of V153I mutation in several studies was measured as follows: Fars Province: about 6\% [4], Isfahan: about 2.04\% [1], and Lorestan: about 2.8\% [16]. The V153I mutation was reported as a common mutation in some studies; however, in the present study, it was reported in only one allele and may not be highly prevalent in deaf individuals in Gilan Province.

In two studies in Shanghai, China [19] and Iran, the G200R variant was introduced as a pathogenic variant [20]. The frequency of this pathogenic variant in Morocco was reported to be almost $0.66 \%$ [21]. In a study in Iran, this pathogenic variant was reported in two alleles with a frequency of $0.05 \%$. This pathogenic variant is in the second quarter (TM4) [22]; its importance in pathogenicity remains unknown. Mahdieh et al. found a small frequency of this variant in more than 10 studies in Iran; however, only in Gilan Province, this pathogenic variant was observed in abundance in an allele.

Due to the high rate of mutation in the GJB2 gene in Rasht and Anzali Cities in Gilan Province, it can be expected that 


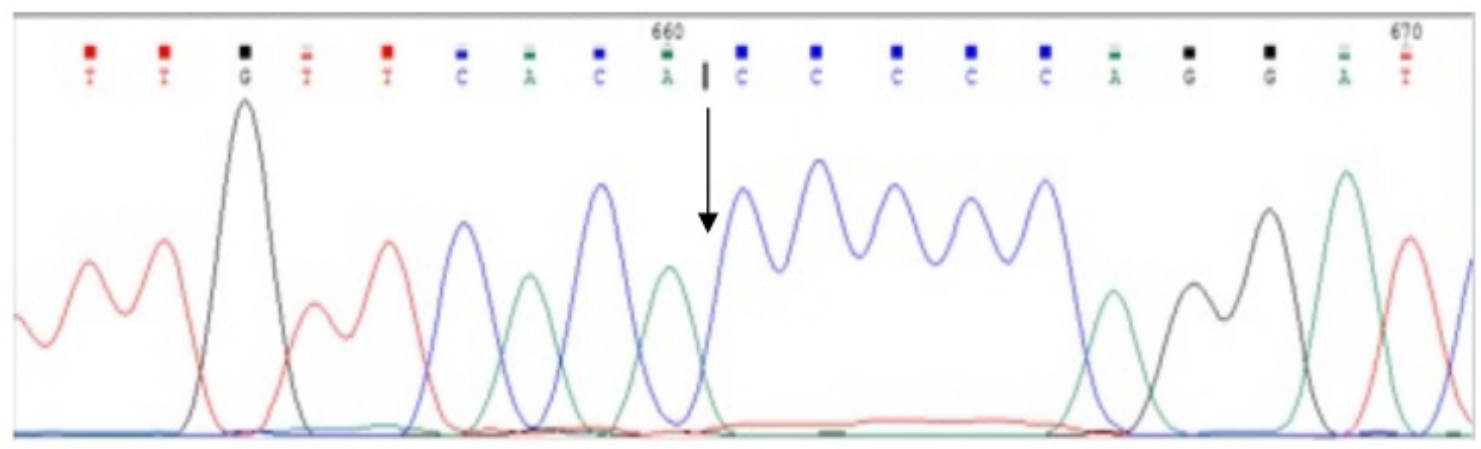

Figure 1. Electrograph of the c.35delG mutation (p.G12VfsX2) as homozygous in a deaf subject

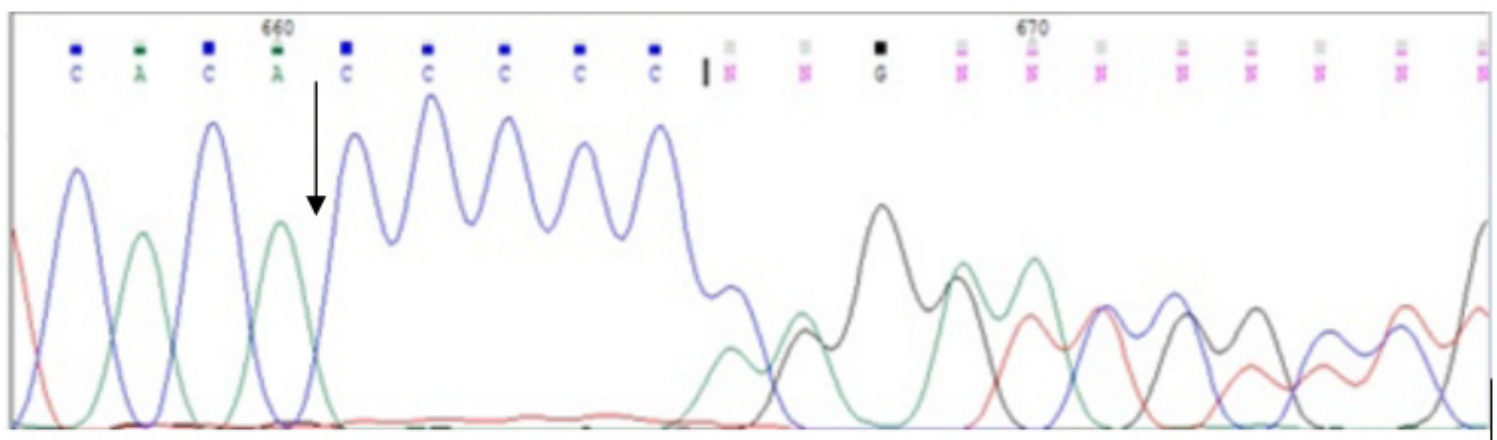

Figure 2. Electrograph of the c.35delG mutation (p.G12VfsX2) as heterozygous in a deaf individual

mutation in this gene is critical in the occurrence of deafness in these cities. Due to the high prevalence of $35 \mathrm{delG}$ mutation concerning the genetic screening of deafness in Guilan Province, it is necessary to study the GJB2 gene as the main gene affecting non-syndromic deafness.

\section{Ethical Considerations}

\section{Compliance with ethical guidelines}

This study was approved by the Ethics Committee of the Islamic Azad University, Tonekabon Branch (Code: IR.IAU. TON.REC.1398.015).

\section{Funding}

This study was extracted from MSc. thesis of the first author at the Department of Genetics, Faculty of Biological Sciences, Tonekabon Branch, Islamic Azad University, Tonekabon.

\section{Authors' contributions}

Conceptualization: Najmeh Ranji and Ali Nazemi; Research and sampling method: Pedram Pouryari Biyachal; Data Analysis, Text Writing, and Review: All Authors.

\section{Conflicts of interest}

The authors declared no conflicts of interest.

\section{Acknowledgements}

We thank the General Department of Welfare of Guilan Province, Welfare of Rasht City, Deaf Centers of Guilan Province located in Rasht and Bandar Anzali, and Mr. Sobhan Darvishi. 


\title{
بروسى جهش هاى ثن GJB2 در اس فرد ناشنواى غيرسندرمى در استان كيلان
}

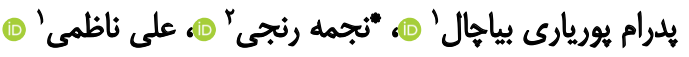 \\ 1. كروه رُنتيك، دانشكده علوم زيستى، واحد تنكابن، دانشكاه آزاد اسلامى، تنكابن، ايران.

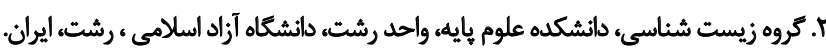

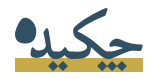

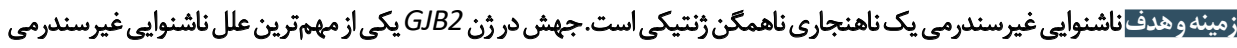

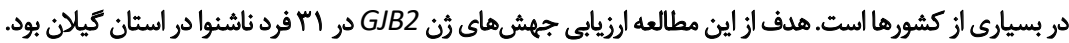

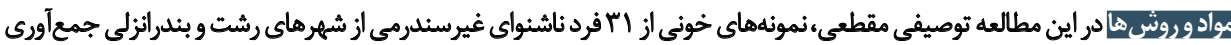

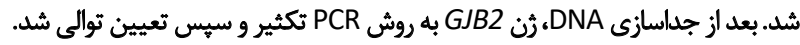

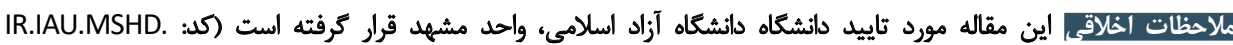
(REC.1398.027

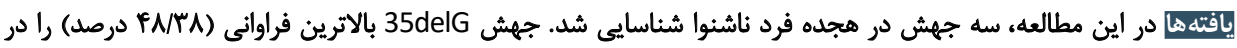

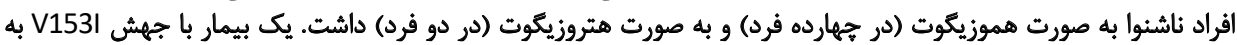

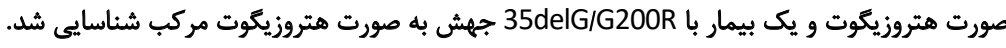

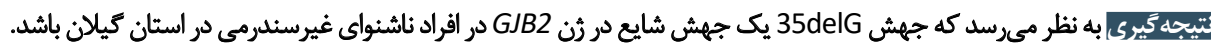

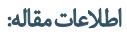

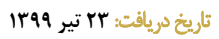

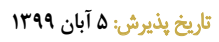

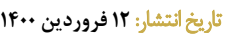

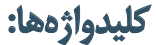

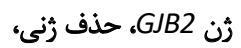
ناشئوايي، توالى يابيى

درصد از موارد ايجاد ناشنوايى غيرسندرمى مىشود [؟].

مقدمه

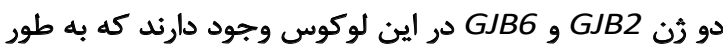

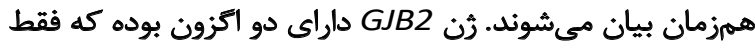

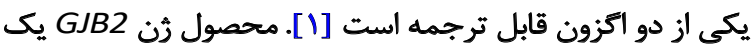

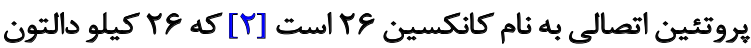
(26kDa)

كانكسين \&ب در كوش داخلى به عنوان يك تنظيم مكنينده

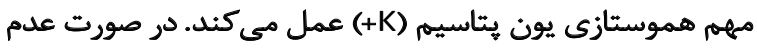

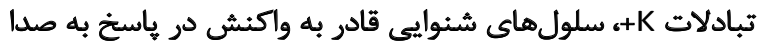

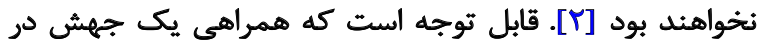

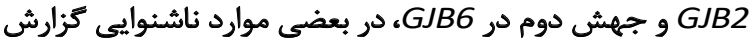

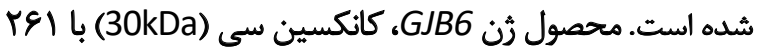

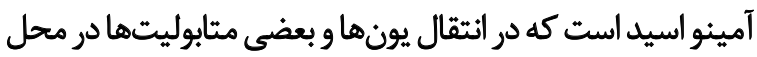
اتصالات سلولهاى شنوايى نقش دارد [1]].

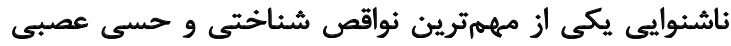

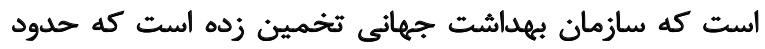

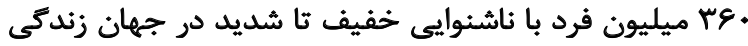

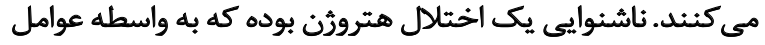
محيطى يا علل رُنتيكى ايجاد مي ئشود [1]

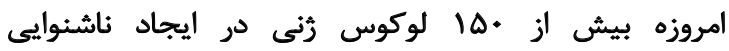

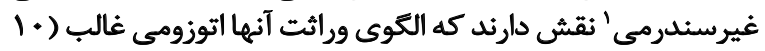

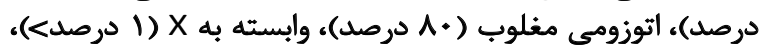

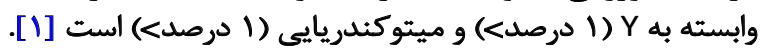

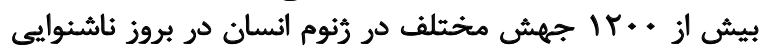
تعيين شده است.

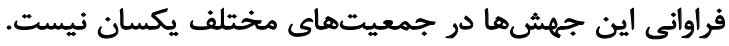

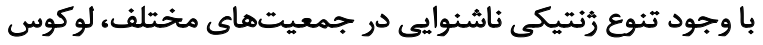

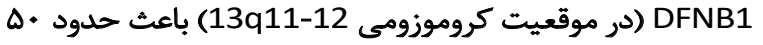

جهش در ثن GJB2، به عنوان مهمثرين علت ناشنوايى ارثى

1. Non-syndromic Hearing Loss (NSHL) 
ناشنوا (با تأييد يزشك معالج موجود در برونده بيمار)، با محدوده

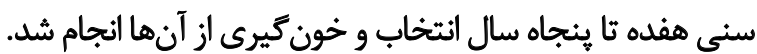
سيس نمونههاى خونى از آس فرد ناشنواى غيرسندرومى

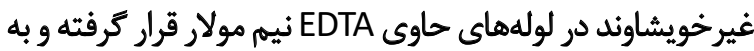

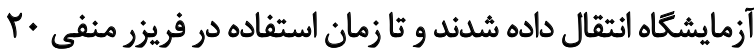
در حالت انجماد نكمهدارى شدند.

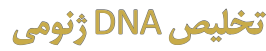

از نمونههاى خونى الب فرد ناشنواى غيرخويشاوند و يك

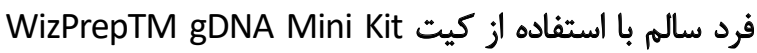

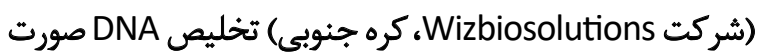
كرفت. جهت اطمينان از تخليص صحيح و سالم بودن

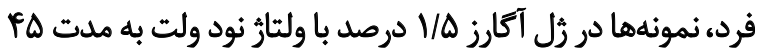

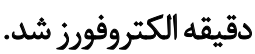

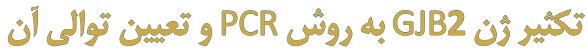

جهت تكثير رن زا GJB2 كيت Pfu master mix (شركت

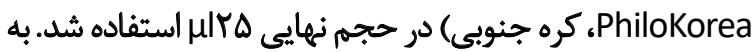

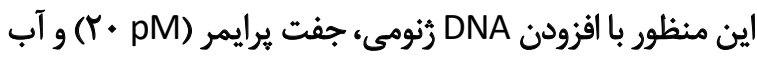

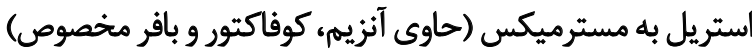
اجزاي واكنش PCR مخلوط شد.

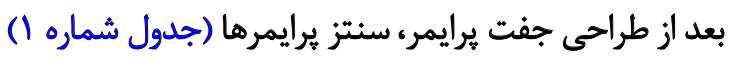

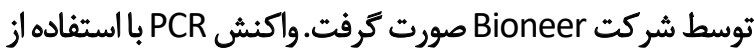
ترموسايكلر Analaytik jena (آلمان) طبق برنامه زير انجام شد: يك مرحله واسرشت شدن ابتدايى در C

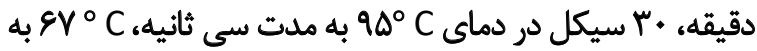

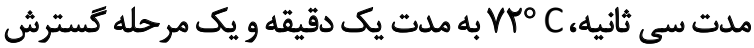

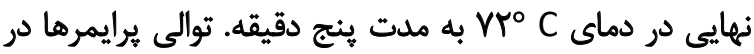
جدول شماره إي ذكر شده است.

بعد از اطمينان از تك باند بودن محصولات PCR با طول باز باز

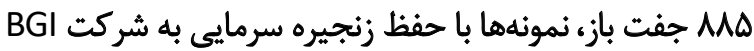
group

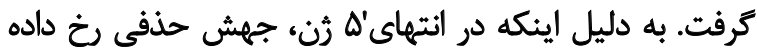

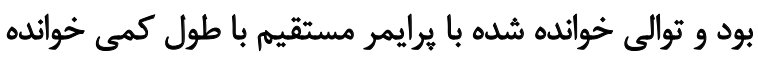

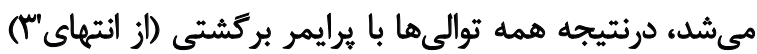

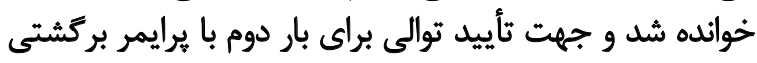
تعيين توالى شد.

نتايج حاصل از تعيين توالى به كمك نرمافزار

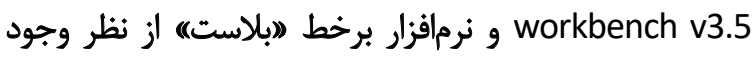

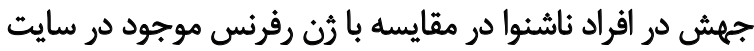

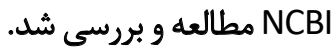

در دئيا شناخته شده است؛ بنابراين بررسى جهشه رهاى اين رثن

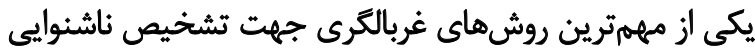

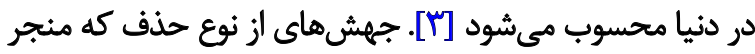

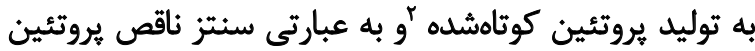

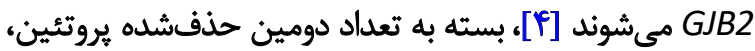

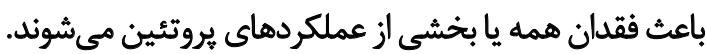
شايعترين جهش در رثن GJB2، جهش

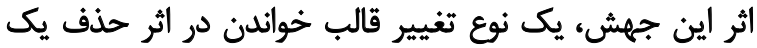

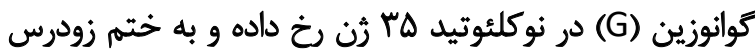

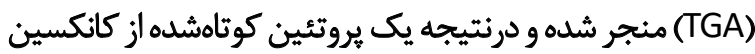

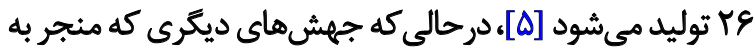

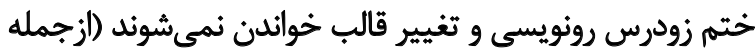

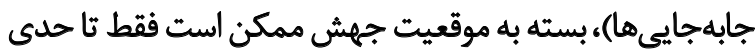

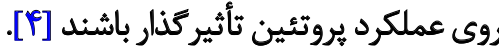

c.35delG بر اساس مطالعات قبلى به نظر مىرسد جهشئ (p.G12VfsX2)

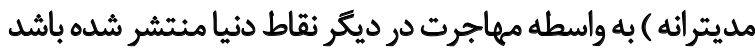

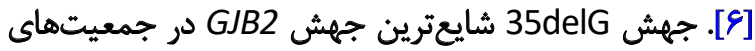

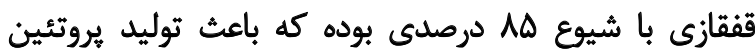

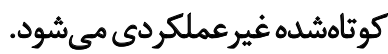

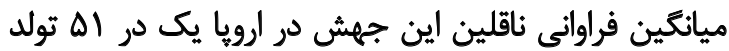

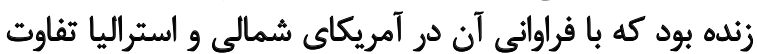

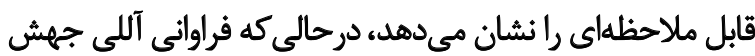

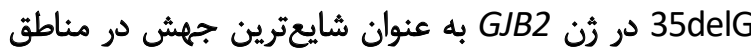

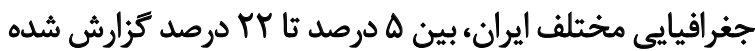

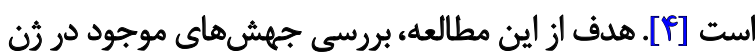

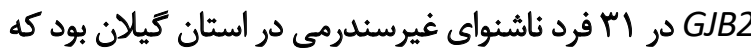
به روش PCR تعيين توالى ارزيابي شد فردا

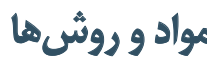

ilyous در اين مطالعه توصيفى مقطعى، در مجموع هم فرد ناشنوا

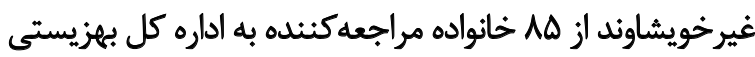

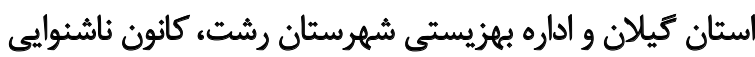

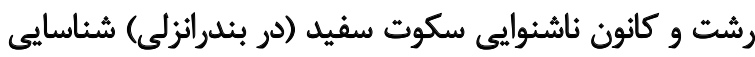
شئله.

اطلاعات بيماران با همكاري اين مراكز و با توجه به اطلاعات

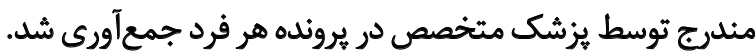

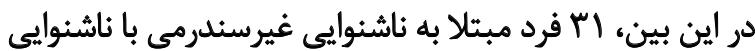

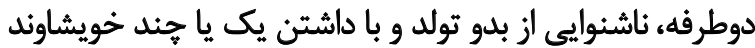


جدول ا. توالى يرايمرهاى مورد استفاده در واكنش PCR

\begin{tabular}{|c|c|c|}
\hline نام برايمر & توالى ثيرايمر & طول محصول \\
\hline GJB2-F & 5'-ATGCTTGCTTACCCAGACTCAG-3' & \\
\hline GJB2-R & 5'-TGTTGGGAAATGCTAGCGACTG-3' & $885 \mathrm{bp}$ \\
\hline
\end{tabular}

هرمزكان حدود \& درصد كزارش شد [ • 1]. در مطالعه جهانكيرى

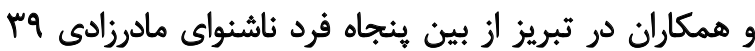

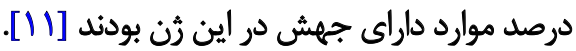
در مطالعه كالاي و همكاران در تركيه شيوع جهش در اين ثرن

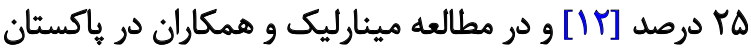

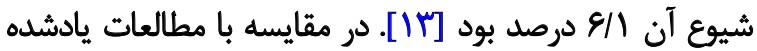

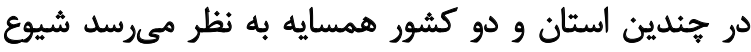

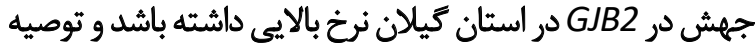

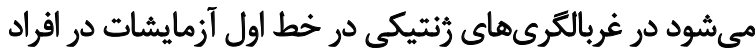
در خطر در اين استان قرار كيرد.

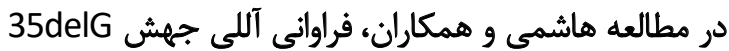

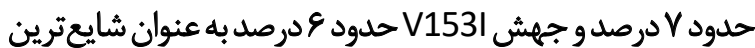

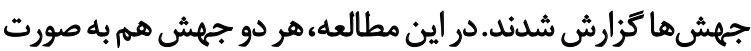

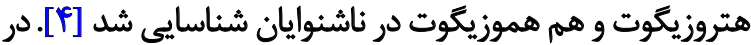

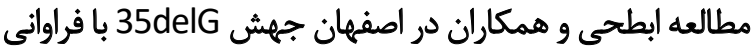

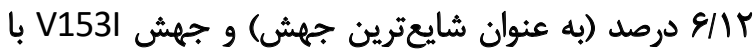

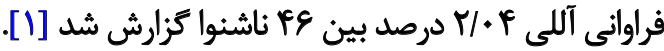
در مطالعه آزادگان و دهكردى، جهش 35delG با فراوانى

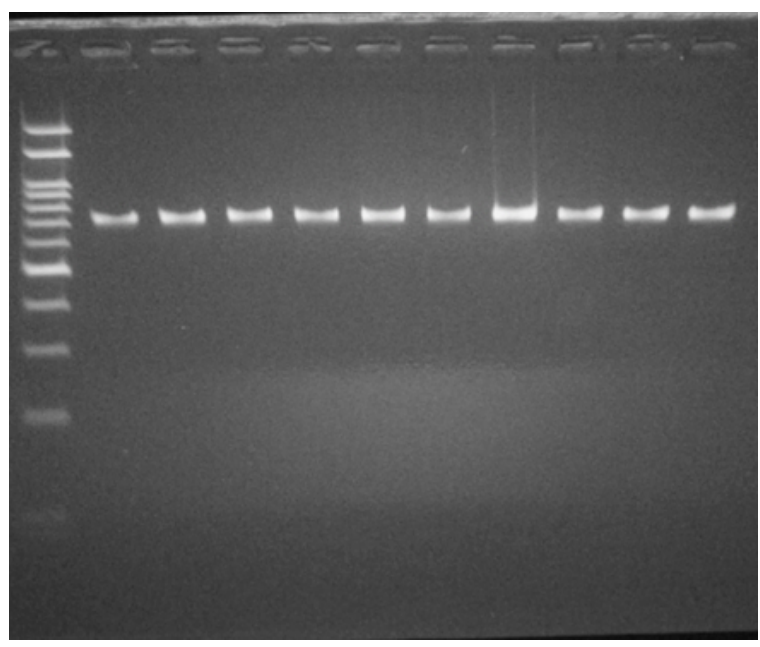

ناشنوايى غيرسندرمى در اثر اختلال در ثرنهاي مختلفى

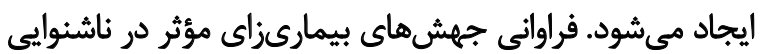

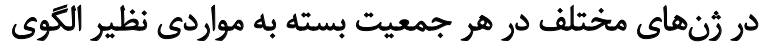

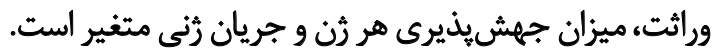

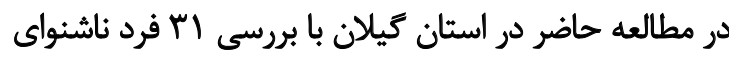

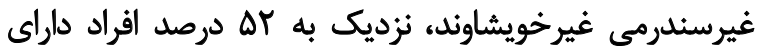

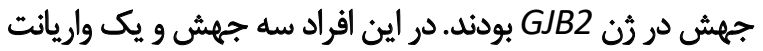

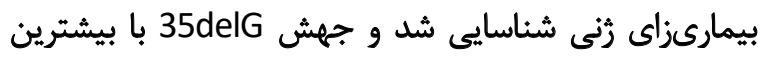
فراوانى در اين مطالعه كَّزارش شئ شد.

در مطالعه هاشمى و همكاران، جهش در رُن GJB2 در در افراد

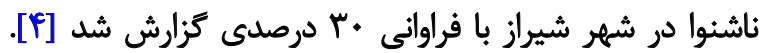

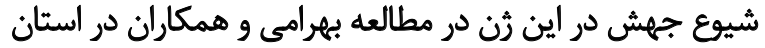

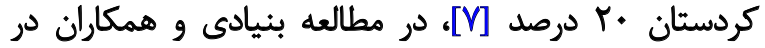

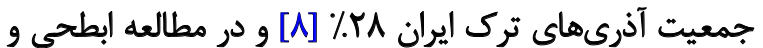

تصوير 1. محصولات PCR مربوط به زن GJB2 در زل آتارز ه/ الدرصد با

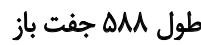
الدر ا .. جفت بازى در جاهك اول از سمت جيب لود شده است.

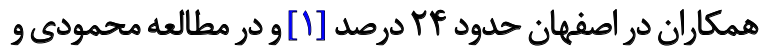

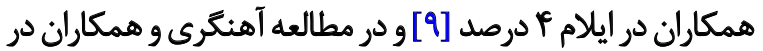




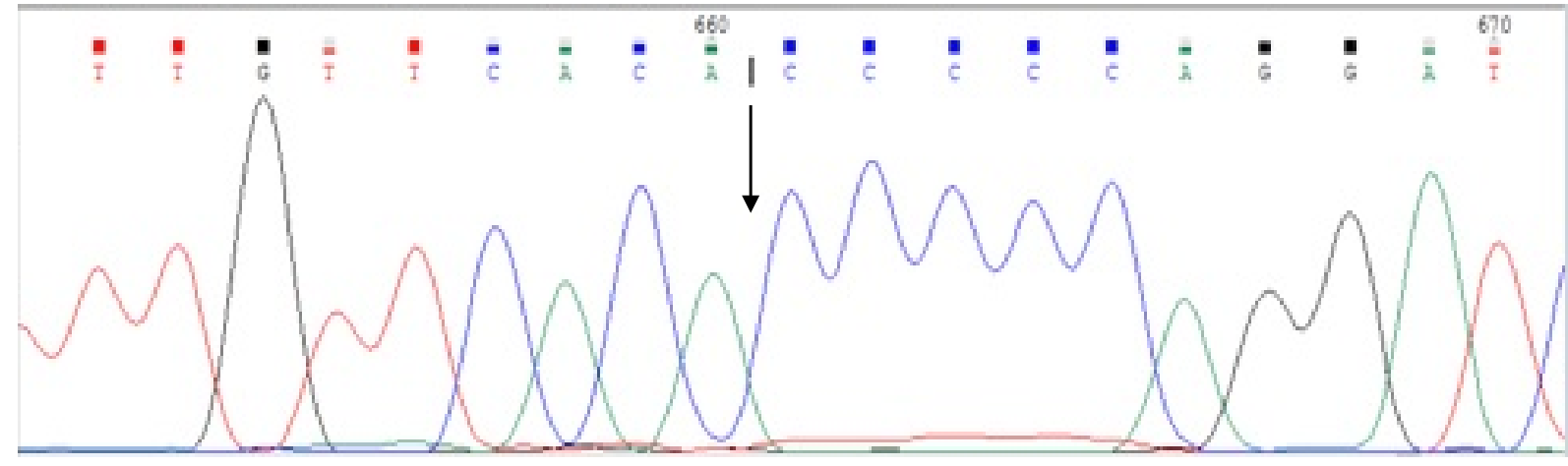

sing

(G12VfsX2.p) 35delG.c به صورت هموزيكوت در يك فرد ناشنوا

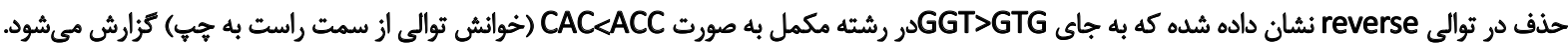

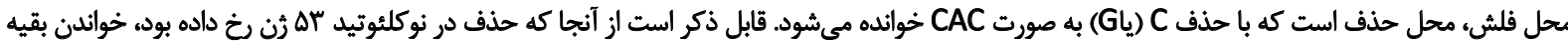

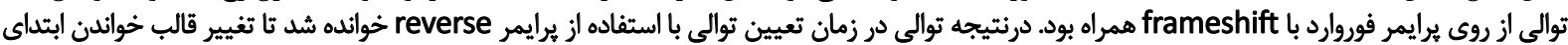

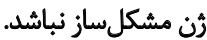

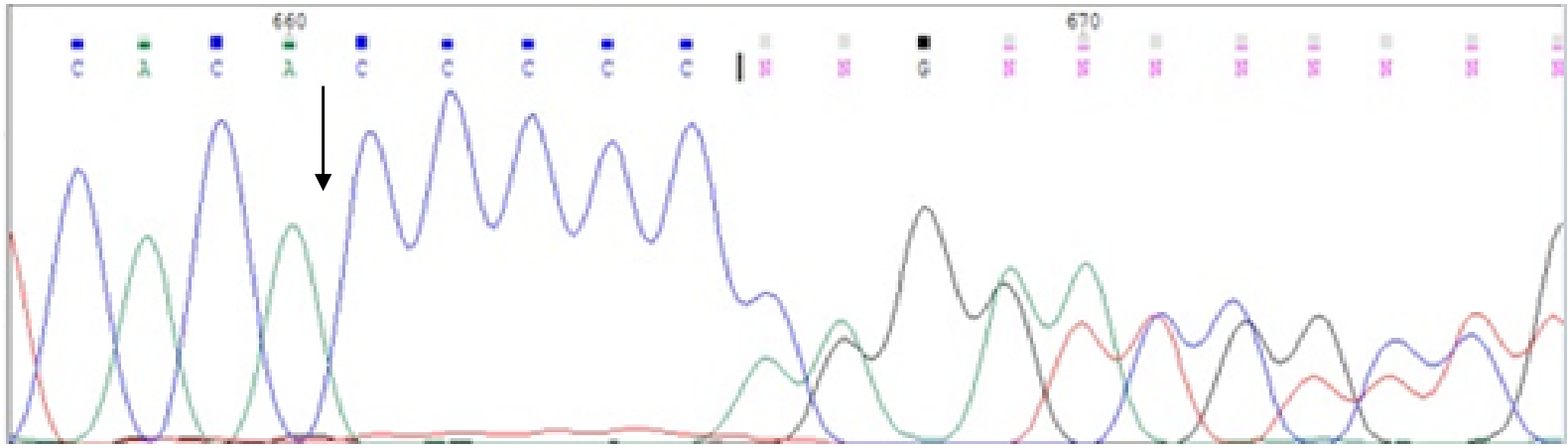

is

تصوير r. الكتروفروكرام مريوط به جهش (G12VfsX2.p) 35delG.c به صورت هتروزيكوت در يك فرد ناشنوا

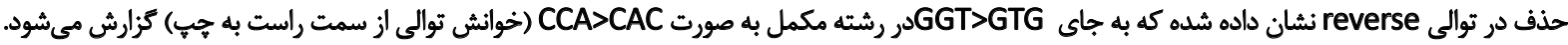
محل فلش، محل حذف است. به دليل هتروزيكوت بودن اختلال در توالى يابي بعد از حذف و وبعد از بئجمين C، به صورت frameshift قابل مشاهده است.

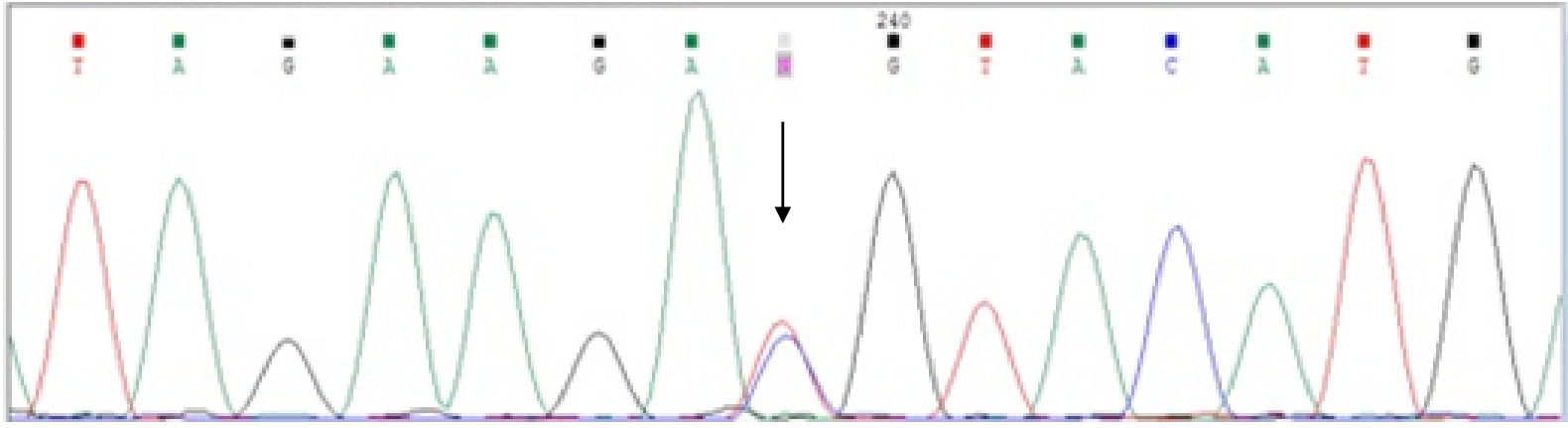

$\sqrt[3]{3}$

تصوير f. الكتروفروكرام مربوط به جهش (V153I.p) A<457G.c به صورت هتروزيكوت در يك فرد ناشئوا

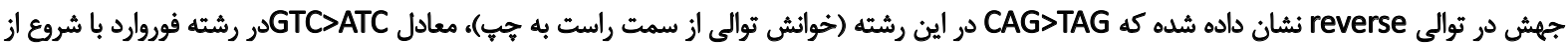
تقطه شروع ترجمه اليت. 


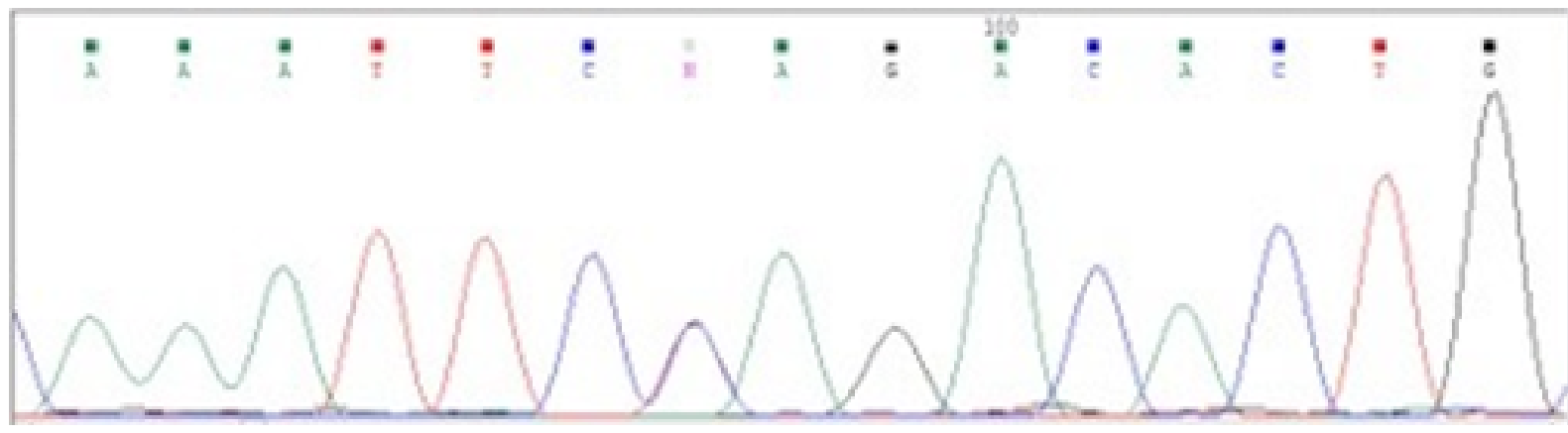

تصوير ه. الكتروفروكرام مربوط به واريانت بيمارىزاي (G200R.p) 598G>A.c بهورت هتروزيكوت در يك فرد ناشنوا

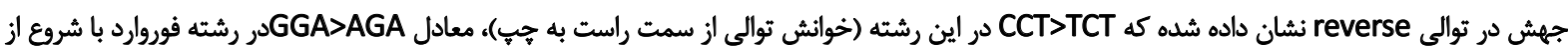
نقطه شروع ترجمه است

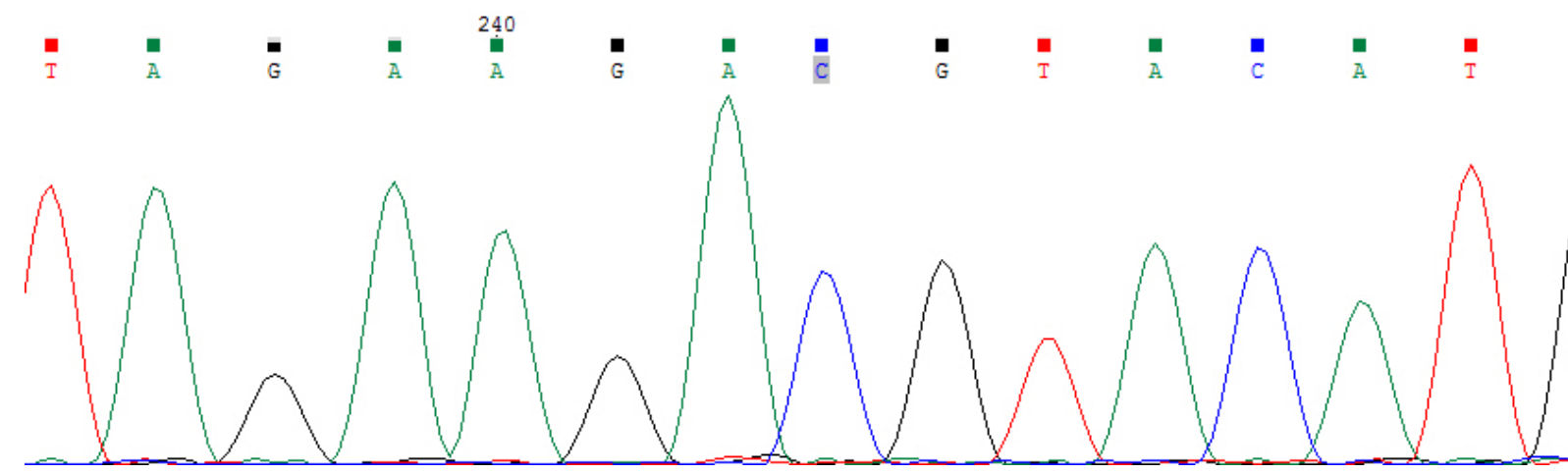

تصوير \&. الكتروفروكرام مربوط به فرد سالم در محدوده كدونى الها در ثن GJB2
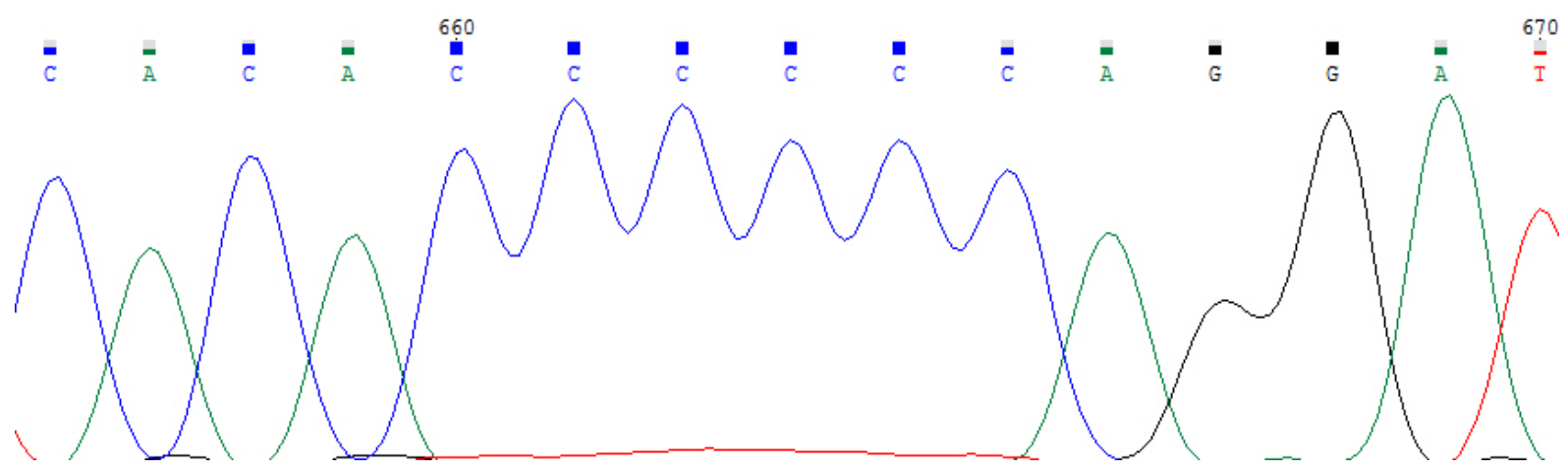

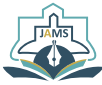

حدود / ح/و درصد موارد داراي جهش

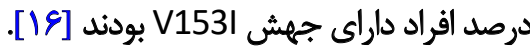

مطالعه حاضر از نظر جهش 35delG بين اس فرد ناشنواى

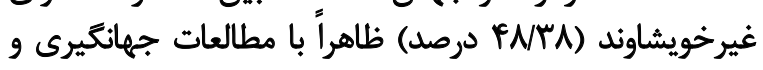

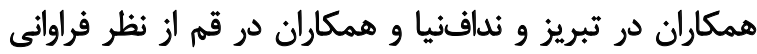

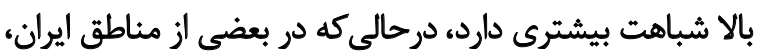

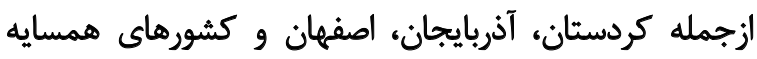

تصوير V. الكتروفروكرام مريوط به فرد سالم در محدوده كدوني به در ثن GJB2

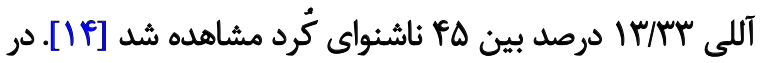

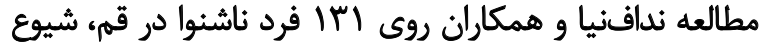

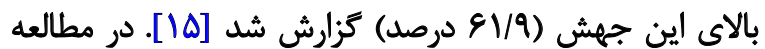

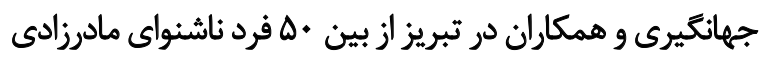

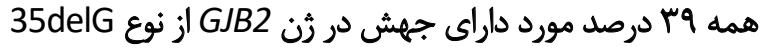

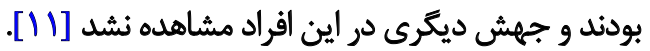
در مطالعه سيهوند و همكاران روى بهه فرد ناشنوا در لرستان 


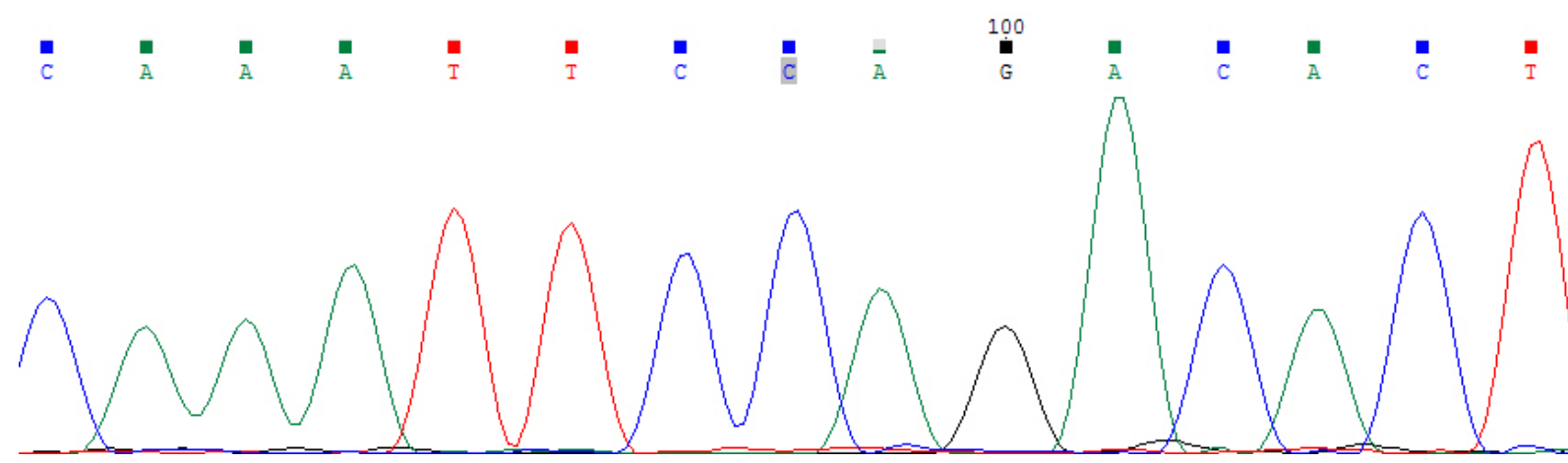

تصوير A. الكتروفروكرام مربوط به فرد سالم در محدوده كدونى r + • در رُن GJB2

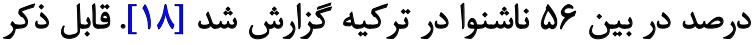

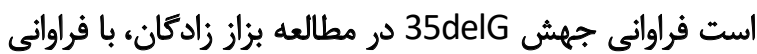

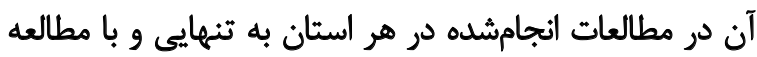

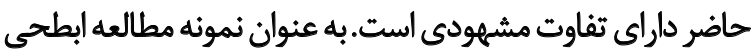
و همكاران در اصفهان جهش

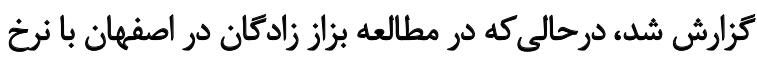

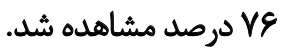

در مطالعه حاضر در استان كيلان جهش 35delG با نرخ

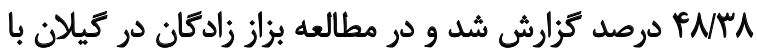

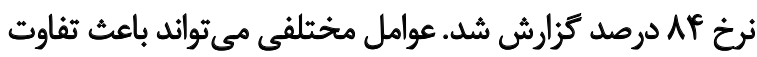

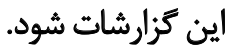

مطالعه حاضر نمونه كيرى از ناشنوايان طى يك دوره شش ماهنه

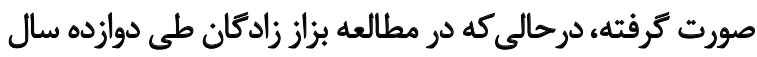
شناسايى افراد ناشنوا در كل كشور صورت در درفت.

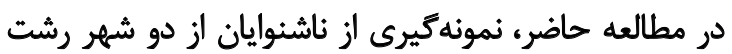

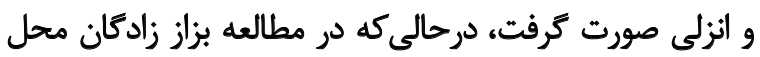

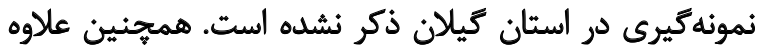

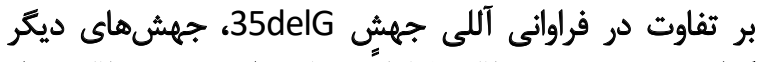

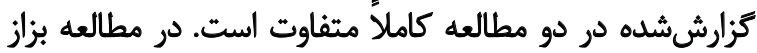

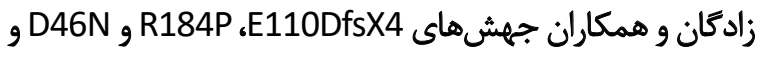

(تركيه و ياكستان) باوجود شايع بودن اين جهش در اين مناطق، ظاهرأ شيوع بايينترى مشاهده شده است

به خاطر نرخ بالاي جهش در اين رُن در شهرهاي مورد مطالعه

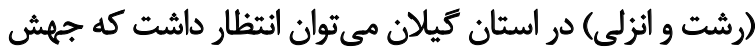

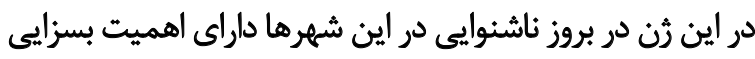

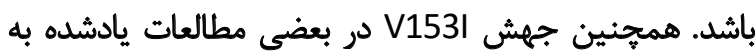

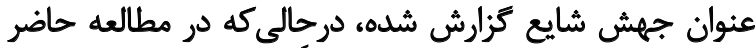

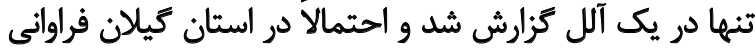
بالايى در بين ناشنوايان نداشته باشد.

از سوى ديكر، در يك مطالعه دوازده ساله توسط بزاز زادكان

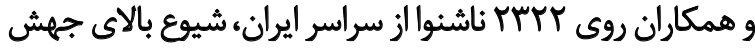

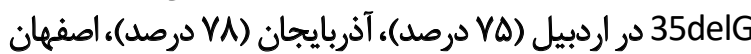

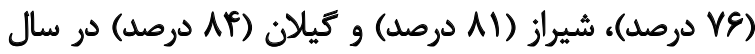

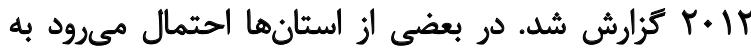

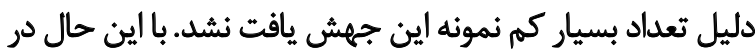

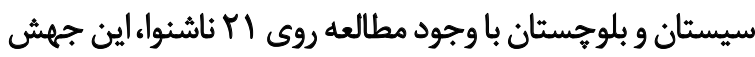

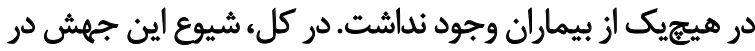
ايران قابل ملاحظه (9F/V درصد) است [IV (IV)]

در مطالعه اييرجى" و همكاران فراوانى آللى اين جهش Ir/f 3. Eyerci

جدول r. جهش هاى مشاهده در رن GJB2 در افراد ناشنوا در استان كيلان

\begin{tabular}{|c|c|c|c|c|c|}
\hline تغييرات بازى & فراوانى نسبي (\%) & تعداد آللها & نوع جهش & 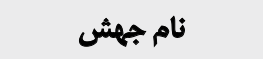 & تعداد بيماران \\
\hline $\begin{array}{l}\text { GGT>GTG } \\
C C A>C A C\end{array}$ & PNIA & r. & حذف تك نوكلثوتيدى با تغيير قالب خوائدن & $\begin{array}{c}\text { c.35delG } \\
\text { p.G12VfsX2 }\end{array}$ & is \\
\hline GTC $>$ ATC & $\mid / 91$ & 1 & جابهجايع بلدمنى & $c .457 G>A$ p.V153I & 1 \\
\hline GGA>AGA & $1 / 91$ & 1 & جابهجايى بلدعنى (واريانت بيماريزا) & $\begin{array}{c}\text { c.598G }>A \\
\text { p.G200R }\end{array}$ & 1 \\
\hline- & $\Delta V / 8$ & $\pi$ & - & - & 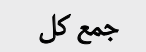 \\
\hline
\end{tabular}


آ فرد ناشنوا با سابقه فاميلى را از بين AD فرد ناشنوا شناسايى

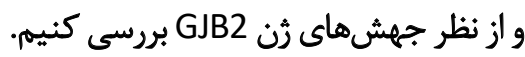

نتيجهيرى

در مطالعه حاضر، حدود ه ه درصد از افراد ناشنوا از كانونهاي ناشنوايى رشت و بندرانزلى در رن ماند

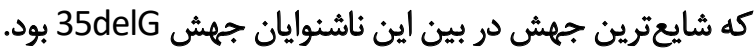

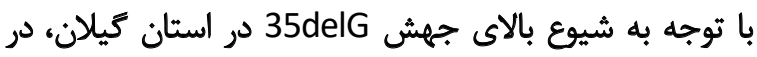

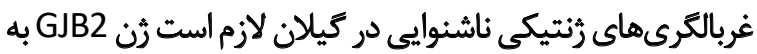

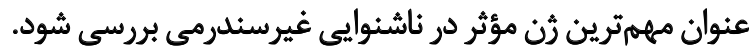

ملاحظات اخلاقى

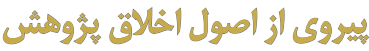

اين مقاله مورد تاييد كميته اخلاق دانشكاه آزاد اسلامى بإي

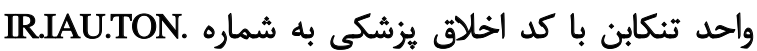
- ه.REC

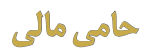

اين مقاله بركرفته از إياياننامه كارشناسى ارشد نويسنده اول در

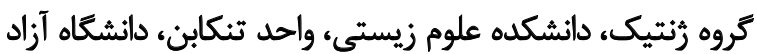

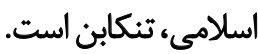

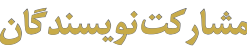

مفهومسازى: نجمه رنجى و على ناظمى؛ روش ئرئ يُوهش و

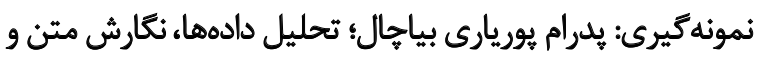

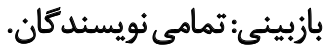

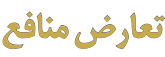

نويسندكان مقاله هيجكونه تعارضى در منافع اعلام نكردند.

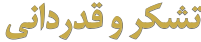

نويسندگًان اين مقاله از اداره كل بهزيستى استان كيلان، بهزيستى شهرستان رشت، كانونهاي ناشنوايى استان كيلان النان

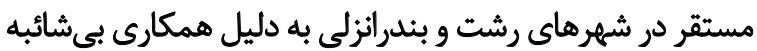

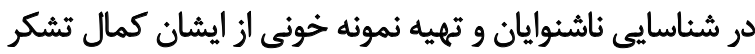

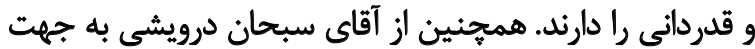

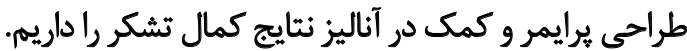

R32C كزارش شد [ [IV]، درحالى كه هيجكدام از اين جهشها در

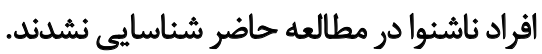

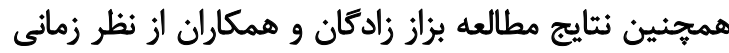

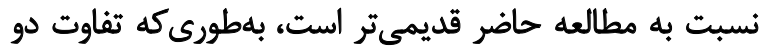

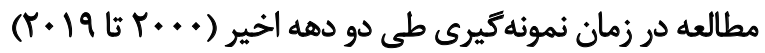

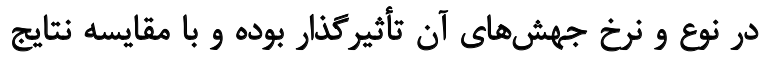

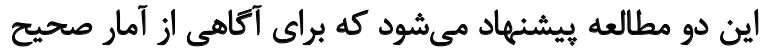

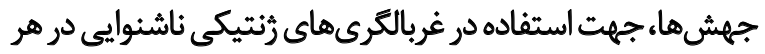

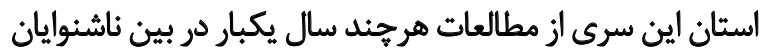

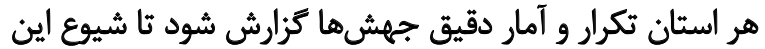
ناهنجارى بين خانوادههاي ايرانى كاهش يابد (IV) مطالعه هاشمى و همكاران در شيراز [أ] و ابطحى و همكاران

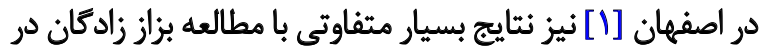

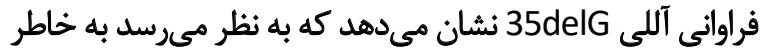

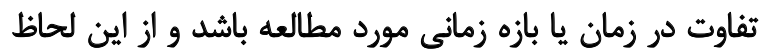

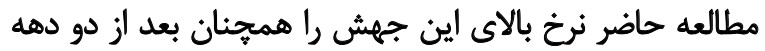

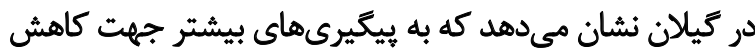

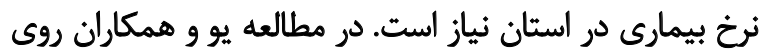

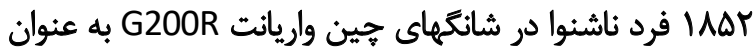

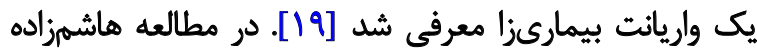
هالشترى و همكاران در تهران و تبرئي

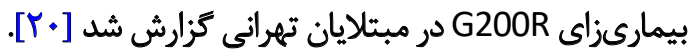

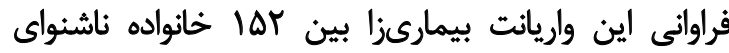

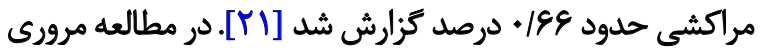

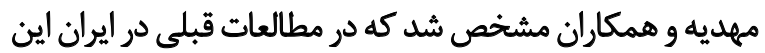

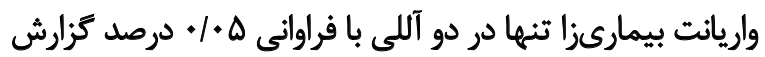

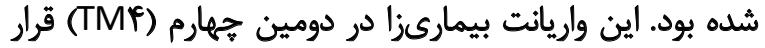

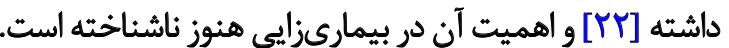

با بينكه مهديه و وهمكاران، فراواني كمى از اين واريانت را داند

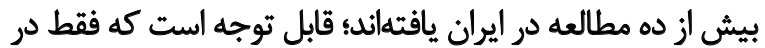

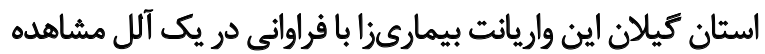

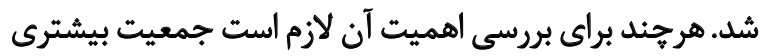

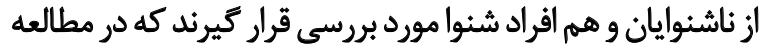
حاضر امكان انجام آزمايشات به اين كستركى وجود نداد نداشت

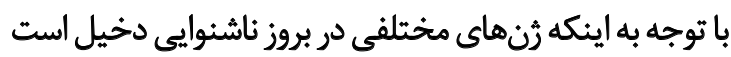

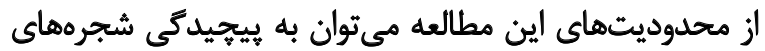

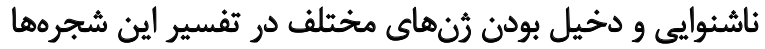

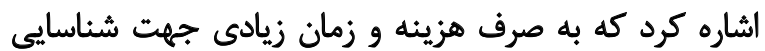
عوامل رُنتيكى بروز اين ناهنجارى نياز دارد.

همجنين أز ديخر محدوديتهاى تحقيق نحوه برقرارى ارتباط

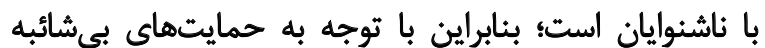

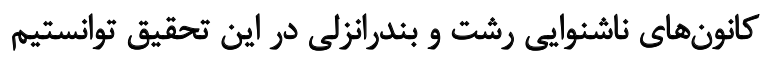




\section{Reference}

[1] Abtahi SHR, Malekzadeh A, Soheilipour S, Salehi M, Taleban R, Rabieian $\mathrm{R}$, et al. Evaluation of GJB2 and GJB6 mutations in patients afflicted with non-syndromic hearing loss. Int J Pediatr 2019; 7(2):9053-60. [DOI:10.22038/IJP.2018.34154.3017]

[2] Amritkumar P, Jeffrey JM, Chandru J, Kalaimathi M, Ramakrishnan R, Karthikeyen NP, et al. Role of DFNB1 mutations in hereditary hearing loss among assortative mating hearing impaired families from South India. BMC Med Genet. 2018; 19:105. [DOI:10.1186/s12881-018-0609-6] [PMID] [PMCID]

[3] Abe S, Nishio SY, Yokota Y, Moteki H, Kumakawa K, Usami SI. Diagnostic pitfalls for GJB2-related hearing loss: A novel deletion detected by Array-CGH analysis in a Japanese patient with congenital profound hearing loss. Clin Case Rep. 2018; 6(11):2111-6. [DOI:10.1002/ccr3.1800] [PMID] [PMCID]

[4] Hashemi SB, Ashraf MJ, Saboori M, Azarpira N, Darai M. Prevalence of GJB2 (CX26) gene mutations in South Iranian patients with autosomal recessive nonsyndromic sensorineural hearing loss. Mol Biol Rep. 2012; 39(12):10481-7. [DOI:10.1007/s11033-012-1929-9] [PMID]

[5] Missoum A. The role of gene GJB2 and connexin 26 in hearing impairment. Ukr Biochem J. 2018; 90(6):5-11. [DOI:10.15407/ubj90.06.005]

[6] Zytsar MV, Barashkov NA, Bady-Khoo MS, Shubina-Olejnik OA, Danilenko NG, Bondar AA, et al. Updated carrier rates for c.35delG (GJB2) associated with hearing loss in Russia and common c.35delG haplotypes in Siberia. BMC Med Genet. 2018; 19:138. [DOI:10.1186/s12881-0180650-5] [PMID] [PMCID]

[7] Bahrami T, Jalilian N, Karbasi G, Noori-Daloii MR. Specific distribution of GJB2 mutations in Kurdistan province of Iran; Report of a relatively isolated population. J Sci Islam Repub Iran. 2017; 28(1):5-11. https:// jsciences.ut.ac.ir/article_59397.htm

[8] Bonyadi M, Esmaeili M, Abhari M, Lotfi A. Mutation analysis of familial GJB2-related deafness in Iranian Azeri Turkish patients. Genet Test Mol Biomarkers. 2009; 13(5):689-92. [DOI:10.1089/gtmb.2009.0026] [PMID]

[9] Mahmoodi H, Mohamadiari S, Sohrab jaidari M, Kordi S, Bakhtiari S, Mahdieh N. [The frequency of mutations in GJB2 gene in deaf subjects referring to the welfare center of llam: Lack of 35delG mutation (Persian)]. J llam Univ Med Sci. 2014; 22(3):41-5. https://www.magiran. com/paper/1299261

[10] Ahangari N, Masoudi M, Poursadegh A, Nejatizadeh A. [Investigating the relationship of genetic mutations in GJB2 and linkage analysis of DFNB4 Locus in a group of non-syndromic hearing impaired people with autosomal recessive inheritance in Hormozgan (Persian)]. Jorjani Biomed J. 2014; 2(2):11-8. http://goums.ac.ir/jorjanijournal/article1-311-en.html

[11] Jahangiri S, Onsori H. [Investigation of the GJB2 gene mutations among subjects with non-syndromic sensorineural hearing loss (Persian)]. Razi J Med Sci. 2017; 24(155):33-9. http://rjms.iums.ac.ir/browse.php?a_ id=4475\&sid=1.\&slc_lang=en

[12] Kalay E, Caylan R, Kremer H, de Brouwer AP, Karaguzel A. GJB2 mutations in Turkish patients with ARNSHL: Prevalence and two novel mutations. Hear Res. 2005; 203(1-2):88-93. [DOI:10.1016/j. heares.2004.11.022] [PMID]

[13] Minarik G, Ferakova E, Ficek A, Polakova H, Kadasi L. GJB2 gene mutations in Slovak hearing-impaired patients of Caucasian origin: Spectrum, frequencies and SNP analysis. Clin Genet. 2005; 68(6):554-7. [DOI:10.1111/j.1399-0004.2005.00529.x] [PMID]
[14] Azadegan-Dehkordi F, Bahrami T, Shirzad M, Karbasi G, Yazdanpanahi $\mathrm{N}$, Farrokhi E, et al. Mutations in GJB2 as major causes of autosomal recessive non-syndromic hearing loss: First report of c.299-300delAT mutation in Kurdish population of Iran. J Audiol Otol. 2019; 23(1):20-6 [DOI:10.7874/jao.2018.00185] [PMID] [PMCID]

[15] Naddafnia H, Noormohammadi Z, Irani S, Salahshoorifar I. Frequency of GJB2 mutations, GJB6-D13S1830 and GJB6-D13S1854 deletions among patients with non-syndromic hearing loss from the central region of Iran. Mol Genet Genomic Med. 2019; 7(7):e00780. [DOI:10.1002/ mgg3.780] [PMID] [PMCID]

[16] Sepahvand M, Kahrizi K, Daneshi A, Riaz Alhosseini Y, Mohseni Marzieh $B N$. [Relative frequency of GJB2 gene mutations in autosomal recessive non-syndromic hearing loss (ARNSHL) patients in Lorestan population (Persian)]. Yafte. 2007; 8(2):89-95. http://yafte.lums.ac.ir/article1-1018-en.html

[17] Bazazzadegan N, Nikzat N, Fattahi Z, Nishimura C, Meyer N, Sahraian $\mathrm{S}$, et al. The spectrum of GJB2 mutations in the Iranian population with non-syndromic hearing loss--a twelve year study. Int J Pediatr Otorhinolaryngol. 2012; 76(8):1164-74. [DOI:10.1016/j.ijporl.2012.04.026] [PMID]

[18] Eyerci N, Altaş E, Pirim I. GJB2 mutations in Turkish patients with nonsyndromic hearing loss. Meta Gene. 2016; 10:56-60. [DOI:10.1016/j. mgene.2016.10.006]

[19] Yu X, Lin Y, Xu J, Che T, Li L, Yang T, et al. Molecular epidemiology of Chinese Han deaf patients with bi-allelic and mono-allelic GJB2 mutations. Orphanet J Rare Dis. 2020; 15:29. [DOI:10.1186/s13023-020-1311-2] [PMID] [PMCID]

[20] Hashemzadeh Chaleshtori M, Hoghooghi Rad H, Dolati M, Sasanfar R, Hoseinipour A, Montazer Zohour M, et al. Frequencies of mutations in the connexin 26 Gene (GJB2) in two populations of Iran (Tehran and Tabriz). Iran J Publ Health. 2005; 34(1):1-7. https://pesquisa.bvsalud.org/ portal/resource/pt/emr-71101

[21] Bakhchane A, Bousfiha A, Charoute H, Salime S, Detsouli M, Snoussi K et al. Update of the spectrum of GJB2 gene mutations in 152 Moroccan families with autosomal recessive nonsyndromic hearing loss. Eur J Med Genet. 2016; 59(6-7):325-9. [DOI:10.1016/j.ejmg.2016.05.002] [P;D]

[22] Mahdieh N, Rabbani B, Wiley S, Akbari MT, Zeinali S. Genetic causes of nonsyndromic hearing loss in Iran in comparison with other populations. J Hum Genet. 2010; 55(10):639-48. [DOI:10.1038/jhg.2010.96] [PMID] 
This Page Intentionally Left Blank 